\title{
Unusual Chief Complaint of Patient with History of Vaccination Leading to Unrecognized Human Rabies with Extreme Risk of Saliva Exposure for Medical Personnel-A Case Report and Literature Review
}

\author{
Kittisak Sripong, M.D.
}

Forensic Unit, Department of Pathology, Faculty of Medicine, Prince of Songkla University, Hat Yai, Songkhla 90110, Thailand. Received 31 August 2019 • Revised 25 November 2019 • Accepted 29 November 2019 • Published online 15 January 2020

\begin{abstract}
:
The aim of this case report is to present an unrecognized case of human rabies with an unusual chief complaint-chest pain-at presentation. The patient had been vaccinated for rabies, presented multiple times at the emergency room, was discharged each time, and was eventually found dead at his house with an unknown cause of death. A post-mortem examination along with a trans-orbital necropsy and real-time reverse transcription polymerase chain reaction test were performed. The results showed rabies virus in the brain tissue.
\end{abstract}

Keywords: chest pain, chief complaint, nasogastric tube insertion, rabies

\section{Introduction}

Rabies is an infectious disease caused by Rabies lyssavirus that occurs in more than 150 countries, according to World Health Organization, "over 95.0\% of human deaths occurring in Asia and Africa". ${ }^{1}$ After the onset of clinical symptoms, it is almost always fatal. ${ }^{1}$ The incubation period of rabies varies ranges from 1 week to 1 year. ${ }^{1}$ There are two forms of the disease- "furious rabies" that manifests with signs of hyperactivity, excitable behavior, hydrophobia, and "paralytic rabies" that manifests with gradual muscle paralysis. ${ }^{1}$ In this case report, the author presents a human rabies case that went unrecognized during multiple hospital visits because of the patient's unusual chief complaint and history of vaccination.
Contact: Kittisak Sripong, M.D.

Forensic Unit, Department of Pathology, Faculty of Medicine,

Prince of Songkla University, Hat Yai, Songkhla 90110, Thailand.

E-mail: skittisa@medicine.psu.ac.th

This is an open access article under the CC BY-NC-ND license

(http://www.jhsmr.org/index.php/jhsmr/about/editorialPolicies\#openAccessPolicy). 


\section{Case report}

A 65-year-old man was found dead at his house by 2 next-door neighbors after multiple emergency room visits for 74.2 hours. This was a forensic case because of an unknown cause of death, which was initiated by legal enforcement agencies requesting a post-mortem examination. The team of officers handling this case comprised a forensic physician and an inquiry official. The overall forensic process started with a post-mortem external examination, followed by the review of the medical records of the deceased, a necropsy, and laboratory investigations.

At the crime scene, numerous dogs were observed barking and running around the nearly invisible area. The navigator leading the team assured us that every dog was vaccinated for rabies. After a few minutes, the house where the body was founded was reached. It did not have a regular entrance; the structure only had an elevated floor and a fence around it that could have prevented dogs from coming in. After climbing into the house, the body was found, and the post-mortem examination began at 00:20 a.m. with inadequate ambient light to conduct the examination. The examination revealed that the body of a thin old man was covered in powder-the face, neck, under the clothes, and both arms and legs. The supravital reaction was positive in the right arm; rigor mortis was observed only in the jaw; livor mortis was detected on the back, which faded with pressure; an abrasion wound size $0.7 \times 0.3$ centimeters was found on the right elbow; and no obvious signs of bite marks were observed (Figure 1). After the post-mortem examination was completed, the exact cause of death could not be determined, so the forensic physician made the decision to take the body to the hospital in order to make further external examinations later in the morning. After conversing with the neighbors, additional information was gained. Before the man died, his breath became shallower, slower and then stopped; no seizures were observed. Moreover, they reported the man had sought medical attention for the health problems leading to his death at a tertiary hospital. Since they had no

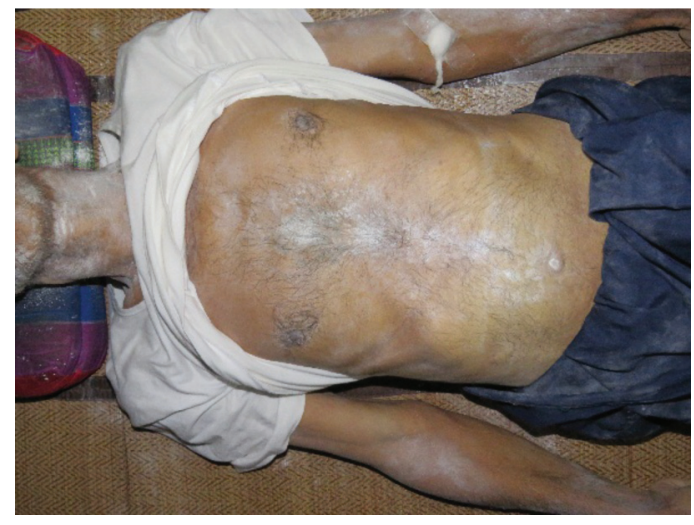

A

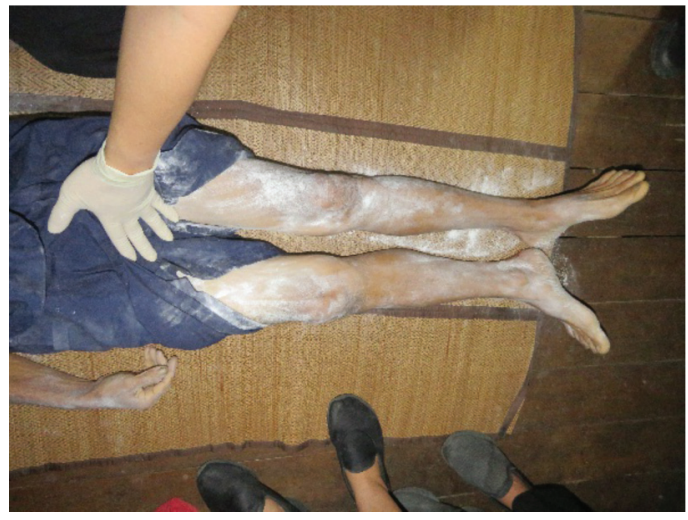

B

Figure 1 Photograph of the deceased

A. Neck, chest, abdomen and both arms covered with powder, along with cotton ball and transpore tape at crook of the left arm

B. Both legs covered with powder 
means to transfer the body to the hospital, a rescue team was called in to assist in the transfer of the body to the morgue of a tertiary hospital where a post-mortem examination was going to be conducted again later that morning.

In the morning, at the Forensic Unit of the tertiary hospital, the medical records of the deceased were reviewed by the forensic physician with the intention to learn more information regarding his underlying diseases or some clue that could lead to the establishment of the cause of death. It was found that he had visited the emergency room 74.2 hours before the time the forensic physician was called for the post-mortem examination; no chronic disease or long history of treatment was found. Each visit was inspected, and their details are described below.

On the first visit at the emergency room, the chief complaint was chest pain for 12 hours. The present illness was determined as chest pain and cough aggravated by drinking; no referred pain, nausea, vomiting, dyspnea, syncope, palpitation or sweating were reported. The past history showed no underlying disease, food or drug allergy, or alcohol consumption-only smoking of 17.5 packs-year. The only abnormal vital sign was a blood pressure (BP) of 190/94 millimeter of mercury $(\mathrm{mmHg})$. The neurological examination showed the patient was alert, his pupils were 2 millimeters in size and reactive to light, and both eyes had full extraocular muscle movements. The electrocardiogram (EKG) showed left ventricular hypertrophy. The chest and lateral neck soft tissue radiograph did not reveal any abnormal findings. The attending physician noted that the patient did not understand the questions regarding the symptoms of chest pain while drinking, so he performed a test by having the patient drink water; it was observed that the patient coughed 2-3 times while drinking. The impression was acute dysphagia and hypertensive urgency; however, since a definite diagnosis of acute dysphagia could not be made, the patient was advised to visit the ear, nose and throat clinic the following day and then discharged from the emergency room.

On the second visit at the emergency room, 4.6 hours after the first visit, the symptoms had worsened; he complained of mid-chest pain with sweating while resting, but no referred pain was reported. Furthermore, he was unable to drink because of the chest pain, but when he managed to drink successfully, he reported not experiencing any dysphagia or odynophagia. He stated he had never had an experience like this before. In addition, he reported having a fever for the past three days, and that the reason for revisiting the hospital was the worsening symptoms. The additional past history information recorded revealed no history of herbal supplement or over-the-counter drug use. Moreover, he had adopted about 80 dogs and reported having been vaccinated for rabies; no history of a dog bite or a dog licking his face was reported. His body temperature of 36.7 degree Celsius. The abnormal physical examination findings were a BP of $201 / 100 \mathrm{mmHg}$, a mildly injected pharynx, and a surgical scar in the abdomen. The neurological examination showed the patient was alert and had a positive gag reflex, a uvula in the midline, no facial palsy, and a motor power of grade $\mathrm{V}$ in all extremities. The EKG showed an additional tall T-wave in the chest lead; however, the serial EKG showed no ST-T change. The chest radiograph showed no pulmonary infiltration or widening of mediastinum. The laboratory investigation finding for Troponin-T was 20.5 nanograms per liter. The diagnosis made was unspecified chest pain; gastrointestinal system dysfunction and psychogenic causes comprised the differential diagnosis list. The initial management consisted of the insertion of a nasogastric tube; the first attempt failed, so the patient was sedated with IV Diazepam 5 milligrams $(\mathrm{mg})$, and the second insertion attempt was successful. The patient was discharged and referred for follow-up for the next 8 days at the internal medicine clinic of the tertiary hospital. 
On the third visit at the emergency room, 34.1 hours after the second visit, the patient presented with nasogastric tube disposition. The abnormal physical examination findings were BP of $199 / 92 \mathrm{mmHg}$, heart rate of 100 beats/ $\mathrm{min}$, and respiratory rate of 24 times/min. The diagnosis of nasogastric tube disposition was made. The management consisted of the re-insertion of the nasogastric tube. The nurse who performed the nasogastric tube insertion failed on three attempts due to a lack of patient co-operation; then the chief resident was called for a fourth try. The chief resident also failed on three attempts; therefore, $5 \mathrm{mg}$ of IV Diazepam were administered, but it was not effective. Finally, the nasogastric tube insertion was successful on the second attempt of a first-year resident. Subsequently, the patient was discharged after receiving appropriate advice.

Eventually, the patient was founded dead at his house, and the forensic physician was called for his postmortem examination at 35.6 hours from his third visit at the emergency room. The overall timeline, pertinent findings, diagnoses, and management at the emergency room are summarized in (Table 1).

Based on the review of the patient's available history presented above, death due to rabies infection was the provisional diagnosis made by the forensic physician. In a situation like this, the forensic management at that time involved the separation of the body with a suspected rabies death from the other bodies in the morgue. Moreover, the morgue officers were informed of the risk for potential infection and advised to protect themselves. The relatives of the deceased were contacted to obtain permission for an autopsy (even though it is not required by law in such a case, it is a requirement based on the well-established practice in the area). The relatives refused permission for the autopsy, so a trans-orbital necropsy was performed using a Tru-cut biopsy needle gauge 20 to obtain 5 specimen pieces; they were stored in a clotted-blood tube, its cap was sealed with paraffin tape, and it was kept at room temperature for transfer to the laboratory as per the relevant guidelines. The Department of Disease Control was called to send the brain tissue for laboratory investigation by airplane to the Neuroscience Center for Research and Development, Bangkok. Next, everyone involved in this case-medical students, inquiry official, emergency room nurses and physicians (especially those involved in the nasogastric tube insertion), hospital porters, laboratory personnel, neighbors, the rescuer and relatives-was notified to warn them about the risk of contracting rabies via secretion exposure. Furthermore, the relatives were advised to not touch the body unless necessary.

Approximately 26.6 hours after the necropsy, rabies virus was detected via the real-time reverse transcription polymerase chain reaction test. The Infection Control Unit of the hospital was alerted officially after that in order to survey the risk of exposure for everyone who had come into contact with the patient.

An outbreak investigation was done, and it resulted in the deceased having been bitten by a stray dog on his left wrist and without a confirmed history of vaccination. ${ }^{2}$

\section{Discussion}

The final diagnosis of rabies after death is not difficult to make; only adequate brain tissue is required for laboratory studies as the guideline suggests. ${ }^{3}$ On the other hand, its clinical diagnosis in patients with unobvious signs and symptoms is very difficult. Especially in the case presented here, the chief complaint of chest pain deviated the medical team's attention/focus away from a diagnosis of rabies to heart disease. Though uncommon, such cases have been reported (Table 2$)^{4-8}$, since rabies can caused focal interstitial myocarditis as discussed by Park et al. ${ }^{9}$ In the case series reported by Mani et al. ${ }^{10}$, all cases presented without evidence of chest pain, so the clinical presentations of rabies vary. Furthermore, concerning the classical sign of fear of water, in our case, the patient 


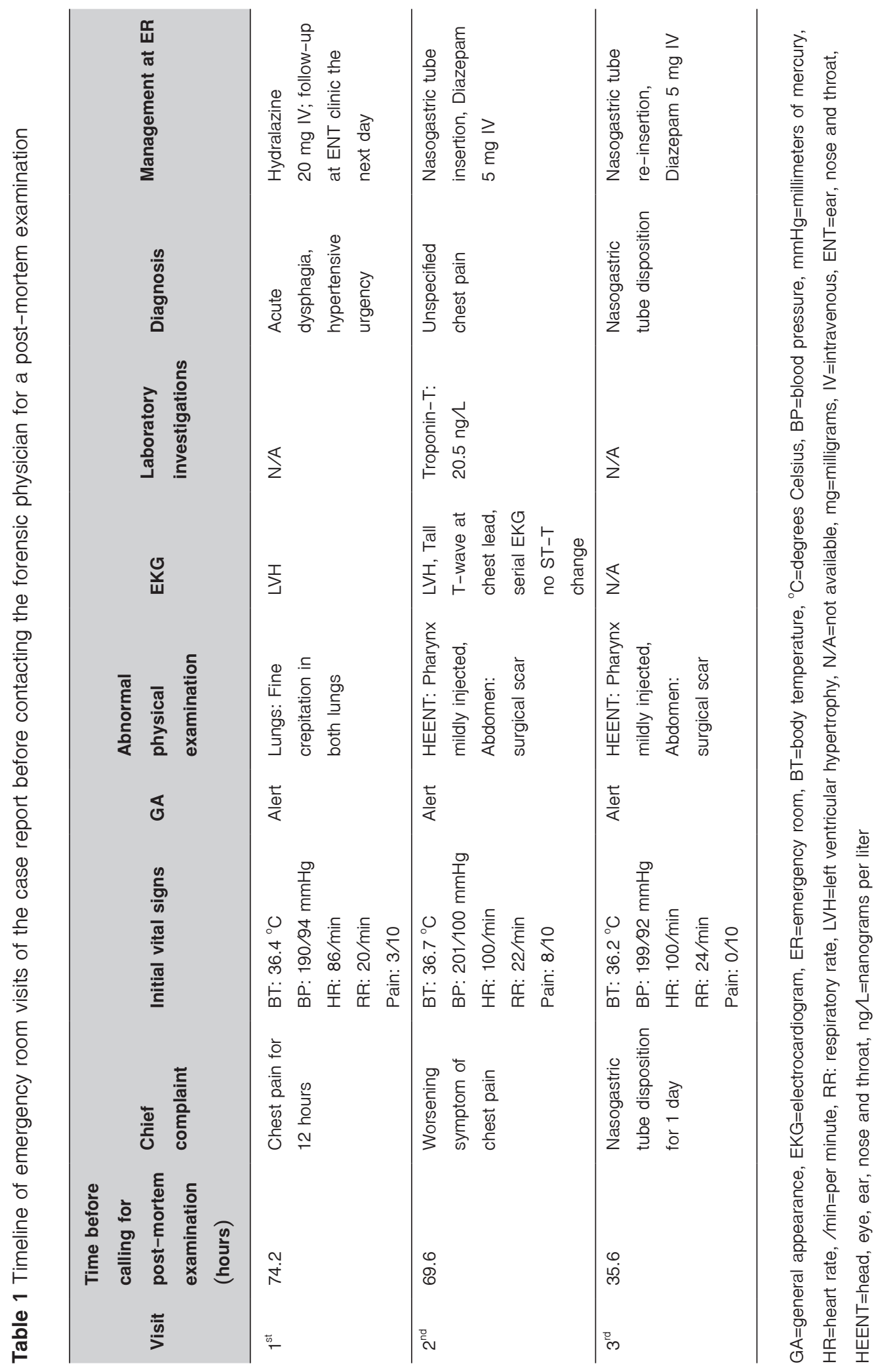




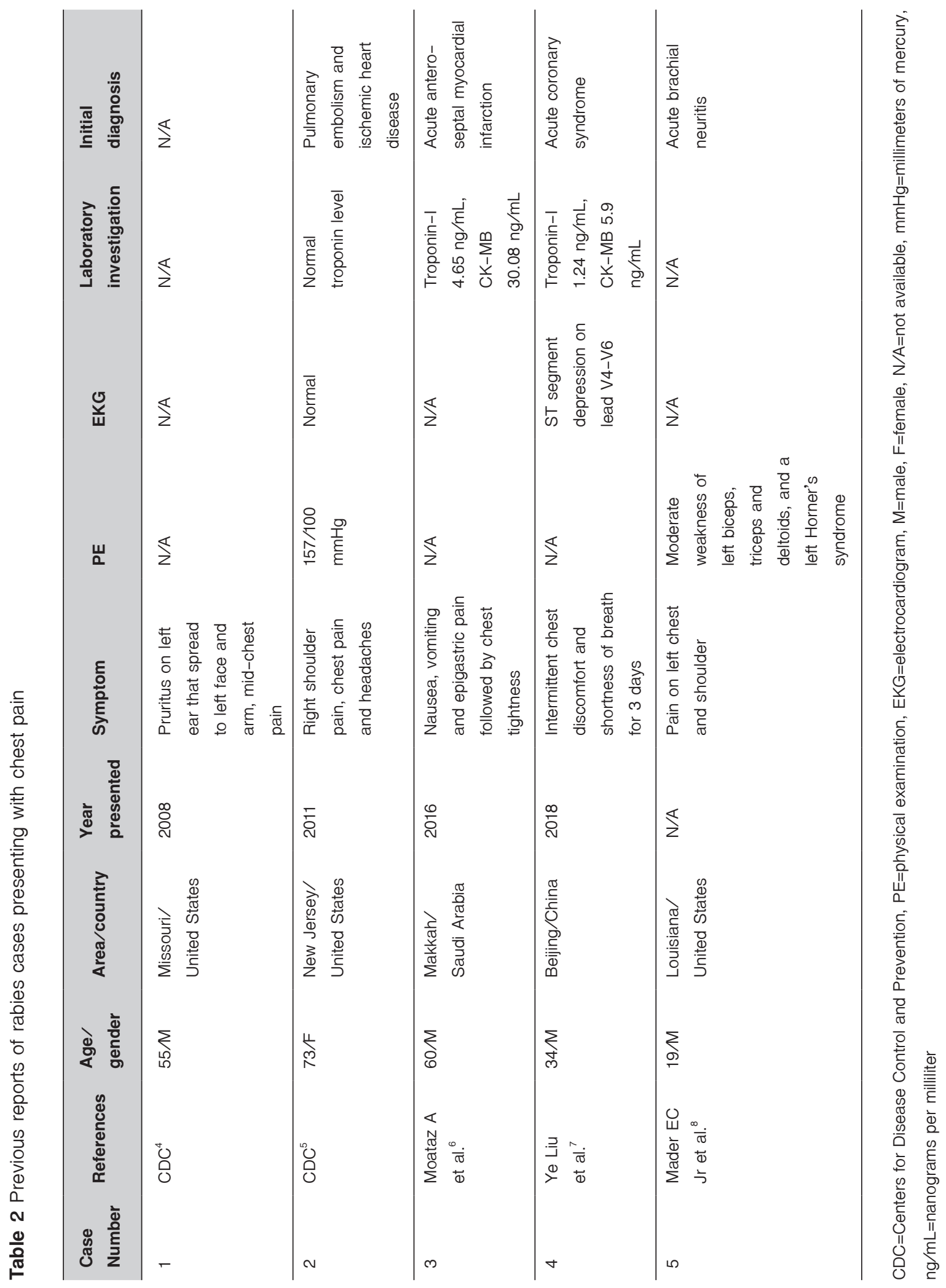


showed that he did not fear water since he could drink in front of the physician. This stands in contrast to the finding of Wertheim et al. that rabies patients experience involuntary inspiratory muscle spasms when presented with a glass of water. ${ }^{11}$

In terms of the forensics aspect, since a rabies investigation is not performed on every case with an unknown cause of death examined by only external examination, the forensic physician thought of rabies infection as the cause of death based on the review of the history recorded on the first emergency room visit and the numerous dogs observed by the forensic physician at the crime scene during the post-mortem visit at the patients residence. Other factors were the fact that he died after an acute onset of symptoms, and that rabies infection is endemic in the province where the deceased resided. ${ }^{12}$ Furthermore, the history of vaccination became irrelevant, since post exposure prophylaxis is not always effective. ${ }^{13}$ Nevertheless, if an autopsy was done, the classical histopathological diagnosis for rabies, "Negri bodies," would have been useful for a conclusive diagnosis; there are reports concerning this in literature involving cases with an unknown cause of death. ${ }^{14,15}$

\section{Conclusion}

Unusual chief complaints must be of concern, especially when suspicious in present illness such as difficulty on drinking reveals. Along with acute coronary syndrome, rabies must be considered in the differential diagnosis. Moreover, the history of rabies vaccination may become irrelevant. As a forensic physician, precaution must be exercised in every case as the deceased may have died from a virulent infectious disease.

\section{Acknowledgement}

The author thanks the Neuroscience Center for Research and Development, Bangkok for the rapid laboratory results. The author also thanks the editors of the International Affairs Office, Faculty of Medicine, Prince of Songkla University for their assistance with editing this paper.

\section{Conflict of interest}

The author declares no conflict of interest.

\section{References}

1. World Health Organization. Rabies [monograph on the Internet]. Geneva: WHO; 2019 [cited 2019 Jul 12]. Available from: https://www.who.int/news-room/fact-sheets/detail/ rabies

2. The Office of Disease Prevention and Control Region 12 Songkhla. Outbreak investigation on the rabies death [monograph on the Internet]. Songkhla: The Office; 2018 [cited 2019 Nov 22]. Available from: https://ddc.moph.go.th/ odpc12/news.php?news $=704$

3. Bureau of General Communicable Disease. Rabies practical guidelines and frequently asked questions. Nonthaburi: Ministry of Public Health; 2016.

4. Centers for Disease Control and Prevention. Human rabies--Missouri [monograph on the Internet]. Georgia: CDC; 2008 [cited 2019 Nov 23]. Available from: https://www.cdc.gov/ $\mathrm{mmwr} / \mathrm{preview} / \mathrm{mmwrhtml} / \mathrm{mm} 5843 \mathrm{a3}$.htm

5. Centers for Disease Control and Prevention. Imported human Rabies-New Jersey [monograph on the Internet]. Georgia: CDC; 2011 [cited 2019 Nov 23]. Available from: https://www. cdc.gov/mmwr/preview/mmwrhtml/mm6051a2.htm

6. Alknawy M, Mohammed I, Ulla SN, Aboud AA. First confirmed case of human rabies in Saudi Arabia. ID Cases 2018;12:29_ 31.

7. Liu $Y$, Yang X, Yang X. Rabies in coronary care unit: a case report. Biomed Res 2018;29:1689-92.

8. Mader EC Jr, Maury JS, Santana-Gould L, Craver RD, El-Abassi $R$, Segura-Palacios $E$, et al. Human rabies with initial manifestations that mimic acute brachial neuritis and Guillain-Barré syndrome. Clin Med Insights Case Rep 2012;5:49-55.

9. Park SC, Crane IM, Pal K, Cagnina RE. Rabies encephalitis myocarditis mimicking ST-elevation myocardial infarction. Open Forum Infect Dis 2019;6:1-3. 
10. Mani RS, Damodar T, S D, Domala S, Gurung B, Jadhav V, et al. Case reports: survival from rabies: case series from India. Am J Trop Med Hyg 2019;100:165-9.

11. Wertheim HF, Nguyen TQ, Nguyen KA, de Jong MD, Taylor WR, Le TV, et al. Furious rabies after an atypical exposure. PLoS Med 2009;6:264-8.

12. Department of Livestock Development. Rabies situation report [monograph on the Internet]. Bangkok: Ministry of Agriculture and Cooperatives; 2018 [cited 2019 Nov 21]. Available from: http://www.dld.go.th/th/images/stories/warning/2561/ 256110/25611012_1rabies.pdf
13. Tinsa F, Borgi A, Jahouat I, Boussetta K. Rabies encephalitis in a child: a failure of rabies post exposure prophylaxis? BMJ Case Rep 2015;1-3.

14. Srinivasan A, Burton EC, Kuehnert MJ, Rupprecht C, Sutker WL, Ksiazek TG, et al. Transmission of rabies virus from an organ donor to four transplant recipients. N Engl J Med 2005; 352:1103-11.

15. Shankar SK, Mahadevan A, Sapico SD, Ghodkirekar MS, Pinto $R G$, Madhusudana SN. Rabies viral encephalitis with probable 25-year incubation period! Ann Indian Acad Neurol 2012;15: 221-3. 